\title{
Application of the connected-moment expansion to single-impurity Anderson Hamiltonians
}

\author{
William J. Massano \\ Department of Science, State University of New York Maritime College, Fort Schuyler, Bronx, New York 10465 \\ Samuel P. Bowen \\ Department of Physics, Virginia Polytechnic Institute, Blacksburg, Virginia 24061 \\ Jay D. Mancini \\ Department of Physics, Fordham University, Bronx, New York 10458
}

(Received 3 August 1988)

\begin{abstract}
We use the connected-moment expansion, recently developed by Cioslowski [Phys. Rev. Lett. 58, 83 (1987)], to investigate the ground-state energy of the single-impurity Anderson model. It is found that the moment expansion obtained for this Hamiltonian is not a Stieltjes series and thus does not provide a useful method for estimating ground-state energies.
\end{abstract}

The study of single-impurity Hamiltonians has, in recent years, been the focus of a great deal of attention. In particular, the Kondo model and the Anderson model have attracted a variety of calculational methods. These include pertubation theory, Hartree-Fock, mean field, renormalization-group theory, Bethe ansataz as well as a number of different variational schemes. Most recently Mancini, Potter, and Bowen ${ }^{1}$ have applied a matrix truncation method that has shown a great deal of promise for estimating the ground-state energy of these systems. Here it was demonstrated that by choosing a truncated basis, with a small amount of effort rapid convergence is achieved and the exact results of the Bethe-ansatz calculations may be approached within a few percent.

Recently, a novel calculational method, the connected-moment expansion (CME), was developed by Cioslowski ${ }^{2}$ and applied the various molecular Hamiltonians. This work was based on a theorem by Horn and Weinstein ${ }^{3}$ concerning the ground-state energy of manybody systems. For any trial ket $\left|\psi_{0}\right\rangle$ the function

$$
\begin{aligned}
F(t) & =\left\langle\psi_{0}|\exp (-t \hat{H}) \hat{H}| \psi_{0}\right\rangle /\left\langle\psi_{0}|\exp (-t \hat{H})| \psi_{0}\right\rangle \\
& =\sum_{k=0}^{\infty}(-t)^{k} I_{k+1} / k !
\end{aligned}
$$

converges to the true ground-state energy $E_{0}$ as $t \rightarrow \infty$. Here the $I_{k}$ are related to the moments $\left\langle\psi_{0}\left|H^{k}\right| \psi_{0}\right\rangle$ of the Hamiltonian. The approach of Horn and Weinstein was to use the method of Padé approximants in order to obtain a viable way of estimating ground-state energies. By looking at when the Padé approximant to the derivative changes sign, they were able to determine at what point the approximation breaks down. Cioslowski was able to avoid the ambiguities involved in using Padé approximants by recasting Eq. (1) into the following form:

$$
F(t)=E_{0}+\sum_{j=1}^{\infty} \alpha_{j} \exp \left(-\beta_{j} t\right) ; \quad \beta_{j}>0
$$

where $a_{j}$ and $\beta_{j}$ are found by direct comparison with Eq.
(2). He then obtained an infinite expansion for the ground-state energy,

$$
E_{0}=I_{1}-\frac{I_{2}^{2}}{I_{3}}-\frac{1}{I_{3}} \frac{\left(I_{4} I_{2}-I_{3}^{2}\right)^{2}}{I_{5} I_{3}-I_{4}^{2}}-\cdots,
$$

where the connected moments $I_{k}$ are defined as

$$
I_{k}=\left\langle\psi_{0}\left|H^{k}\right| \psi_{0}\right\rangle-\sum_{i=1}^{k-2}\left[\frac{k-1}{i}\right] I_{k+1}\left\langle\psi_{0}\left|H^{k-i-1}\right| \psi_{0}\right\rangle .
$$

Cioslowksi points out that the construction of the connected-moment expansion, Eq. (3), is easy due to the straightforward nature of the recursion. Also having the property that is size extensive makes the connectedmoment expansion attractive for many-body calculations.

Knowles ${ }^{4}$ has presented thorough critique of the CME for molecular electronic structure calculations and has pointed out a number of possible limitations to its use. He showed that an improved series may be obtained by considering a generalized Padé approximant to $F(t)$ provided that the recast expansion $F(t)$ is a Stieltjes series. This will be the cure provided that the Hadamard determinants constructed from the $I_{k}$ 's are positive. In general it is unknown whether $F(t)$ is a Stieltjes series and hence whether the series will converge. If, however, the first negative moment $I_{k}$ appears for large enough $k$ there is hope that a reasonable estimate to the ground-state energy may be obtained.

In this work we wish to investigate the validity of the connected-moment expansion for a class of Hamiltonians which describe a single impurity embedded in an otherwise perfect host lattice. The well-known Anderson model is used as a test case for this method. We have chosen our trial ket to be the filled Fermi sea $\left|\psi_{0}\right\rangle=|F\rangle$ since it is known that the true ground state of these systems is a singlet. Also, for calculational efficiency we have chosen $|F\rangle$ to have zero energy.

(c) 1989 The American Physical Society 


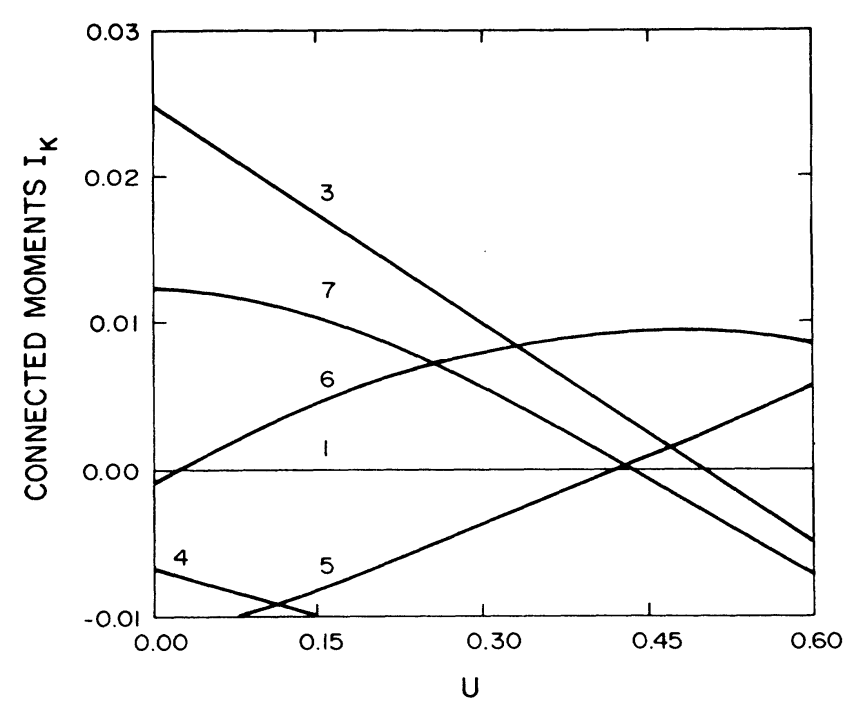

FIG. 1. The connected moments $I_{k}$ are plotted vs the Coulomb energy $U$. Each curve is labeled by the index $k$. $I_{2}=0.1$ and is not shown.

The Anderson Hamiltonian is given in second quantized form by

$$
\begin{aligned}
H= & \sum_{k, s} \varepsilon_{k s} n_{k s}+U N_{\uparrow} N_{\downarrow}+\sum E_{f} N_{f} \\
& +\frac{v}{\sqrt{N}} \sum_{k, s}\left(c_{k s}^{\dagger} f_{s}+f_{s}^{\dagger} c_{k s}\right)
\end{aligned}
$$

where the usual notation is used. The operators with a $k$ subscript refer to the host conduction electrons while the subscript $f$ refers to the impurity site. The parameter $U$ represents the on-site Coulomb repulsive energy and $V$ is the hybridization energy.

In Fig. 1 we have plotted six of the first seven connected moments of the Anderson model. The number next to each curve represents the index of that connected moment. The second moment is constant and has the value +0.1 and would be off the graph. All of these connected moments are plotted as a function of the Coulomb energy $U$ over the range $0.0<U<0.6$, in units of $\pi \Delta$.

Note that even for $U$ very small the connected moment $I_{4}$ is negative and as the Coulomb energy increases many

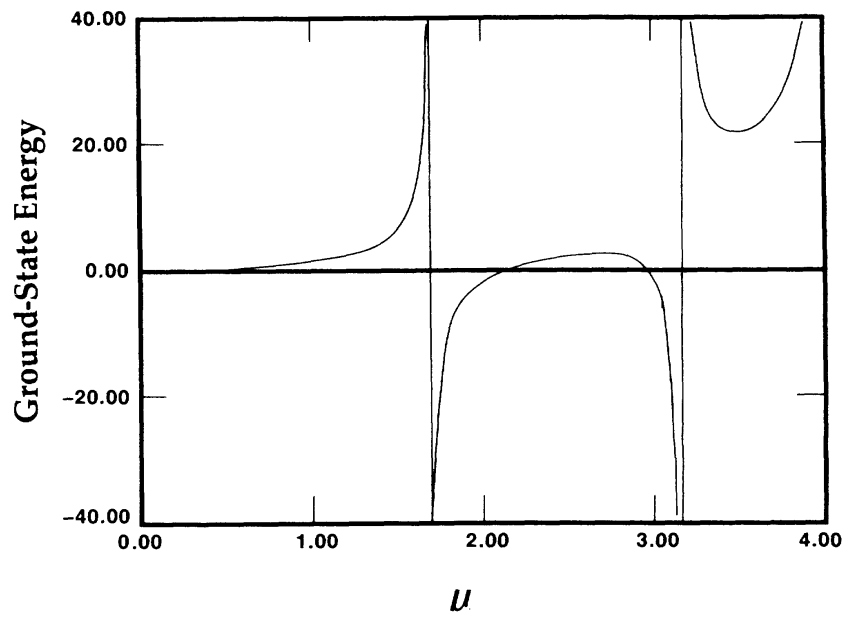

FIG. 2. The ground-state energy estimated by Eq. (3) is shown as a function of the Coulomb energy $U$. Note the very unstable behavior.

of the connected moments also pass through zero. For this Hamiltonian the sequence of $I_{k}$ for $k>4$ is not a Stieltjes series for small $U$ and this becomes worse for larger $U$.

In Fig. 2 we plot the best ground-state energy estimate that can be found from a knowledge of the first seven moments of the Hamiltonian and Eq. (3). As can be seen from this figure the ground-state energy behaves very wildly as the Coulomb integral $U$ is changed.

The expressions for the first seven moments were evaluated by a symbolic manipulation program that was tailored for this type of Hamiltonian and has been checked against analytic calculations. The ground state of this same Hamiltonian has been calculated using these same moments, but using a direct Lancsos method ${ }^{5}$ and no such instabilities were found.

The discontinuities evident in Fig. 2 stem from the zeros of the denominator in the CME approximants. However, it is not clear what the physical basis is for the instabilities shown for this model Hamiltonian. It is clear, however, that the application of the connected moment expansion may be more restricted than first realized.
1J. D. Mancini, C. D. Potter, and S. P. Bowen, J. Appl. Phys. 61, 3168 (1987).

${ }^{2}$ J. Cioslowski, Phys. Rev. Lett. 58, 83 (1987); Phys. Rev. A 36, 374 (1987); Chem. Phys. Lett. 136, 515 (1987); Phys. Rev. A 36, 3441 (1987).
${ }^{3}$ D. Horn and M. Weinstein, Phys. Rev. D 30, 1256 (1984).

${ }^{4}$ P. J. Knowles, Chem. Phys. Lett. 134, 512 (1987).

5 J. D. Mancini, W. J. Massano, C. D. Potter, and S. P. Bowen, Phys. Rev. B 38, 5047 (1988). 